\title{
Los Learning centres en el Reino Unido. Estudio de caso de seis universidades medianas.
}

\author{
Por Rocío Serrano-Vicente
}

\begin{abstract}
Resumen: El objetivo de este estudio era investigar si las bibliotecas universitarias que pertenecen a universidades británicas de pequeño y mediano tamaño están adoptando el concepto de centro de recursos para el aprendizaje, y qué consecuencias se han derivado desde el punto de vista de los servicios, espacios y estructura del personal. Se investigó también la provisión de recursos electrónicos, especialmente el desarrollo de plataformas de enseñanza virtual. Interesaba conocer si todos los cambios llevados a cabo en los últimos años eran debidos a una mejora del apoyo al aprendizaje. Se estudiaron también las estructuras organizativas y los servicios con el objetivo de conocer cómo se están desarrollando y si el cambio de servicios y de edificios está afectando el apoyo a la investigación.
\end{abstract}

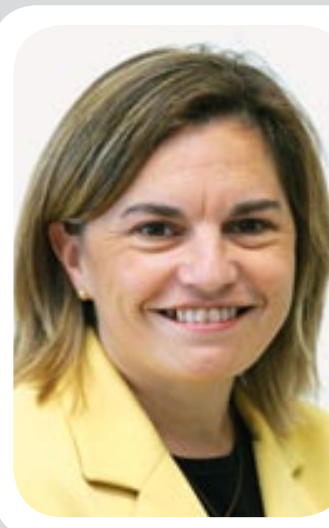
Rocío Serrano es diplomada en biblioteconomía y licenciada en documentación por la Universidad de Extremadura. Durante el curso 2003-2004 realizó el master in librarianship en la Universidad de Sheffield (UK). Ha trabajado en la gestión de revistas electrónicas en la biblioteca de ciencias de la Universidad de Navarra. Actualmente es subdirectora del Servicio de Bibliotecas de esa universidad. Ha investigado en temas relacionados con el aprendizaje y la biblioteca universitaria.

Palabras clave: Bibliotecas universitarias, Apoyo al aprendizaje, Convergencia, Apoyo a la investigación.

\section{Title: Learning centres in the United Kingdom: a case study of six mid-sized universities}

Abstract: The aim of this study was to investigate whether academic libraries at small and medium-sized British research universities are adopting the Learning Centre concept and determine the consequences from the point of view of services, space allocation and personnel structure. Provision of electronic resources in these libraries is described, especially the development of Virtual Learning Environments. Organizational structure and user services are also detailed, illustrating how structures are evolving and the possible impact of changes in services and the physical plant on support for research.

Keywords: University libraries, Learning support, Convergence, Research support.

Serrano-Vicente, Rocío. “Los Learning Centres en el Reino Unido. Estudio de caso de seis universidades medianas”. En: El profesional de la información, 2007, julio-agosto, v. 14, n. 4, pp. 307-318.

DOI: 10.3145/epi.2007.jul.04

\section{Introducción}

Se exponen los resultados de un estudio realizado durante el año 2004 en algunas bibliotecas universitarias del Reino Unido. El objetivo era investigar si las bibliotecas pertenecientes a instituciones de pequeño y mediano tamaño orientadas a la investigación estaban aplicando el modelo de Centro de Recursos para el Aprendizaje y en su caso, cómo afectaba a los servicios, espacio y estructura del personal.

Para comprender mejor el contexto es necesario conocer algunos datos de la historia reciente de las universidades del Reino Unido. En 1992 hubo un cambio en las instituciones de educación superior británicas. La consecuencia más importante fue que desapareció la distinción entre las universidades y los llamados "politécnicos", que antes no tenían categoría de universida- des y donde se impartían estudios de carreras más prácticas. Desde ese momento, estos últimos se empezaron a llamar "nuevas universidades" (Naylor, 1997). En ellas la investigación no está tan desarrollada, siendo la enseñanza el primer objetivo de los profesores. Para las "universidades antiguas", sin embargo, la investigación es vital; los fondos económicos para llevarla a cabo dependen de los resultados de la evaluación de la investigación que se realiza periódicamente (research assessment exercise, $R A E$ ). Por lo tanto, hay una presión más intensa respecto a los resultados en estas universidades, por lo que requieren una provisión de materiales y un entorno más adecuado de apoyo a la investigación.

En 1993 se publicó el informe Follett del Libraries Review Group. Intentaba resolver algunos problemas que afectaban a las bibliotecas universitarias: un mayor 
número de estudiantes, desarrollo de las nuevas tecnologías y recursos financieros estancados dentro de las instituciones de educación superior (Beton, 1999). Incluía algunas recomendaciones para implementar nuevos entornos para edificios de universidades, acompañadas de fondos económicos que ayudaron a construir nuevos edificios (Joint Funding Councils' Libraries Review Group, 1993; Brophy, 2000; Edward; Fisher, 2002).

El informe Dearing (National Committee of Inquiry into Higher Education, 1997) encargado con la finalidad de determinar una serie de recomendaciones para mejorar la educación superior, enfatizaba "la necesidad de desarrollarse en una sociedad 'que aprende' con la finalidad de competir en el mercado mundial". Ante la introducción de las nuevas tecnologías, la información cobra una mayor importancia, y es necesario que los nuevos titulados posean habilidades en el uso, búsqueda y recuperación de información, lo que implica cambios en el modo de enseñanza y aprendizaje (Beton, 1999). El documento insiste en la importancia del papel que las bibliotecas deben jugar en las actividades de enseñanza en la universidad (Brophy, 2001). En esta línea, la construcción del Centro de Recursos para el Aprendizaje se ha visto como una forma de llevar a cabo las recomendaciones de los informes Follet y Dearing.

Se utilizan varios términos en inglés para denominar estos centros, siendo los más comunes: Learning Resource Centres y Learning Centres. También se conocen, sobre todo en EUA, con el nombre de Information Commons o Learning Commons. En este trabajo hemos utilizado el nombre de Learning Centre ( $L C$ ), pues es el que comúnmente se está utilizando en el Reino Unido ${ }^{1}$.

Graham Bulpitt (2003) define el Learning Centre como: "un entorno dinámico que integra la provisión de recursos para apoyar una amplia variedad de actividades de aprendizaje tanto individuales como en grupo". Un centro de recursos es un lugar donde los estudiantes van a encontrar apoyo en su estudio: el entorno es propicio para aprender y estudiar. Es una mezcla de biblioteca y servicios de información, ordenadores y materiales multimedia y educacionales. La idea es apoyar y mejorar la calidad docente y en algunos de estos centros, incluso ayudar a los profesores en las tareas de enseñanza (Oyston, 2003a).

El presente estudio analiza cómo se ha aplicado el concepto de $L C$ en algunas "universidades antiguas" de pequeño y mediano tamaño, teniendo en cuenta que el apoyo a la investigación es una parte importante de su misión. La razón de seleccionar específicamente este tipo de universidades se debe a que, principalmente, el modelo de $L C$ se había aplicado en las nuevas universidades más que en las antiguas.
"La experiencia acumulada en el Reino Unido, donde llevan más de una década con este modelo, puede ser de interés para las bibliotecas universitarias españolas"

En primer lugar se envió un cuestionario a las seis bibliotecas que participaron en el estudio con el fin de obtener algunos datos cuantitativos. Se realizaron después entrevistas semiestructuradas para tener una visión más profunda de ellas. Por último se llevaron a cabo visitas a los edificios para tomar algunos datos más de la observación.

Se presenta por tanto un estudio realizado en un número muy reducido de universidades. En principio interesaba centrarse en aquellas más orientadas a la investigación de pequeño y mediano tamaño. Existe un grupo en el Reino Unido que las reúne, llamado el "1994 group", compuesto por 19 universidades, pero sólo siete de ellas respondieron en un primer momento, de las que seis contestaron al cuestionario. En cualquier caso, se aportan datos de la instalación y su funcionamiento, así como opiniones de los directivos, lo que hace que este estudio sea aplicable a las bibliotecas universitarias españolas, muchas de las cuales se encuentran en la actualidad en un proceso de adaptación al modelo de $L C$ en sus instalaciones. La experiencia acumulada en el Reino Unido donde llevan más de una década con este modelo puede ser de interés.

Los centros estudiados fueron Universidad de Bath, Reading, Sussex, Birkbeck College (University of London), Royal Holloway (University of London) y Universidad de York ${ }^{2}$.

\subsection{Metodología empleada: cuestionarios y en-} trevistas semiestructuradas

Se enviaron primero cuestionarios por correo electrónico a las bibliotecas participantes que incluían ocho preguntas: dos se referían a la estructura organizativa, tres al edificio y otras tres al cambio de servicios en los últimos años.

Una vez recibidas las respuestas se elaboraron las entrevistas, eligiéndose un formato semiestructurado con un doble objetivo. Por una parte, se pretendía que los datos resultantes pudieran ser comparables entre las bibliotecas. Pero también se deseaba dar cierta libertad al entrevistado para que pudiera exponer sus propias opiniones y enfoques sobre los diversos aspectos. Además, al conocer ciertos detalles de cada centro se pudo orientar la entrevista hacia aquellos puntos que parecían más interesantes en cada uno de los casos. 

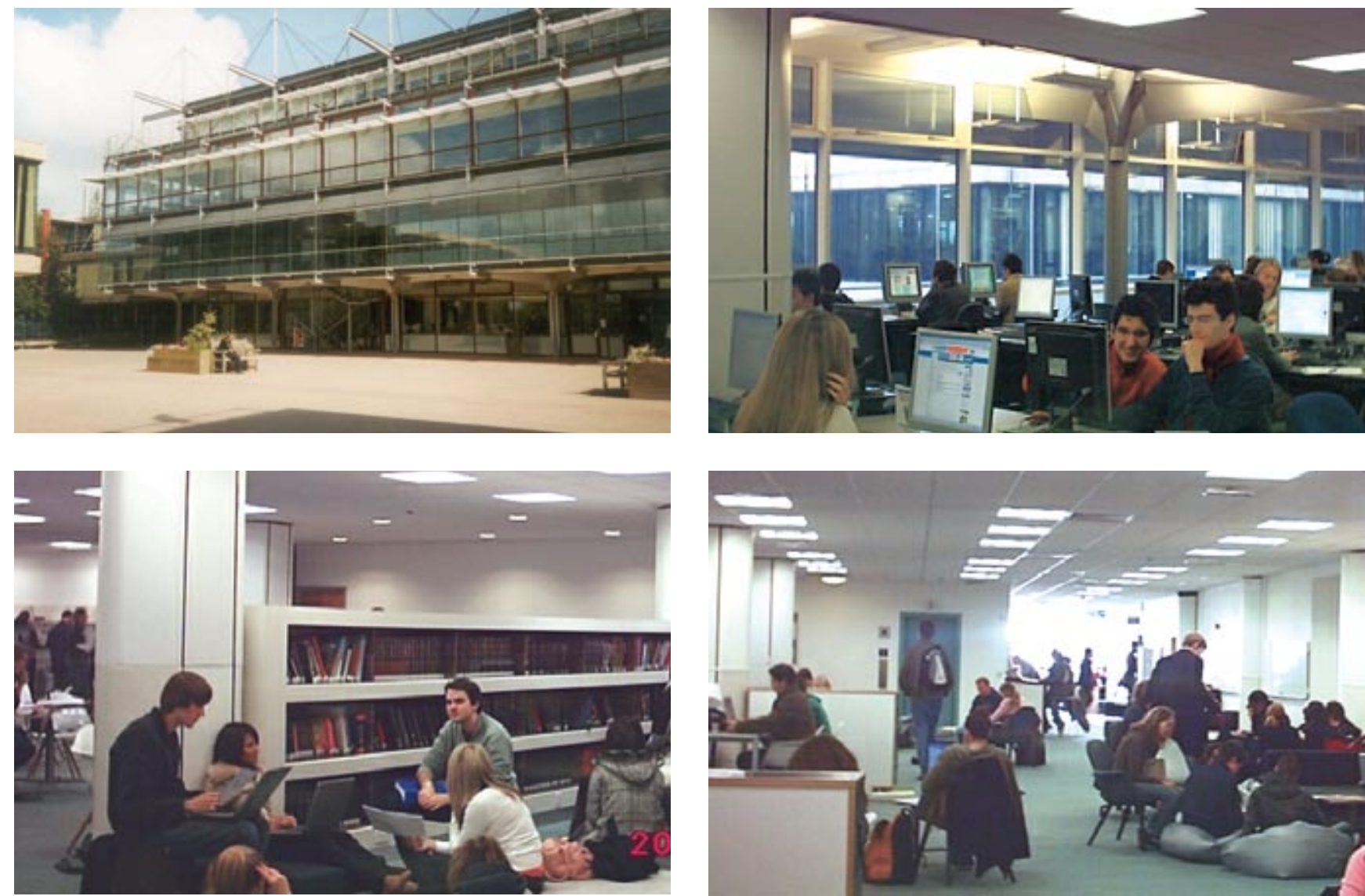

Imágenes del Learning Centre de Bath

Hay que señalar que los entrevistados se ofrecieron muy gustosamente a participar en el estudio, y que aportaron no sólo datos sobre las bibliotecas, que fueron muy interesantes, sino valoraciones personales que ayudaron mucho al desarrollo de la investigación. Una vez obtenidos, y junto con las visitas a las instalaciones, se codificaron y estudiaron, comparándolos entre sí para poder sacar conclusiones a la luz de la revisión bibliográfica realizada.

\section{Edificio}

Los cambios en el tipo de enseñanza y aprendizaje han contribuido a hacer del $L C$ un nuevo tipo de edificio, diferente de la biblioteca tradicional; su misión es diferente así como su propósito. Como afirma Hines (2003), el edificio está directamente relacionado con las actividades educativas, no solamente con la situación de los recursos. De hecho, los cambios en éstos o en los servicios que ofrece el $L C$ son pequeños: la diferencia está en cómo interactúan.

"Los cambios en el tipo de enseñanza han contribuido a hacer del LC un nuevo tipo de edificio, diferente de la biblioteca tradicional y con una nueva misión"
Frecuentemente las universidades que adoptan el modelo del $L C$ construyen nuevas instalaciones, tratando de reflejar esa filosofía a través del nuevo entorno físico, aunque no siempre es posible. Por eso, las bibliotecas en muchas ocasiones tienen que conformarse con adaptar sus edificios al nuevo entorno de apoyo al aprendizaje mediante una nueva distribución, que incluya espacios para trabajo en grupo, o añadiendo ordenadores y otros medios.

\subsection{Entorno físico para el aprendizaje}

Los bibliotecarios deben facilitar las actividades de enseñanza e investigación, no sólo pensando en el presente, sino también en el futuro (McDonald, 2000). La diferencia principal con la biblioteca académica tradicional es que el edificio está directamente relacionado con las actividades pedagógicas (Hines, 2003).

Los alumnos aprenden no sólo individualmente, sino también a través del entorno social. La diferencia radica en que además ahora este espacio educativo pertenece a los alumnos, no a los profesores. McDonald (2004) reconoce que en el pasado, en las bibliotecas y centros de recursos se enfatizaba en los puestos de lectura, nuevas tecnologías y un amplio abanico de medios audiovisuales, más que en las tradicionales colecciones impresas. Más recientemente, los nuevos $L C$ proveen espacios para el aprendizaje informal, lugares para trabajo en grupo y para el uso de ordenadores portátiles. 
Sin embargo, como señala Hines (2003), lo que hace distinto a este tipo de centros no son los recursos o servicios que se ofrecen, sino cómo ambos interactúan. En este sentido, la distribución abierta, los espacios para trabajo en grupo, y para estudio en silencio, por ejemplo, transmiten fácilmente la filosofía del $L C$ cuando se entra en el edificio.

\subsection{Cómo se ha aplicado la filosofía del $L C$}

Las respuestas a los cuestionarios señalan que todas las bibliotecas han realizado un cambio o remodelación de sus edificios (tabla 1). Sin embargo, los resultados de la investigación indican que hay pocas que hayan adoptado en su totalidad el modelo de $L C$ (Bath y Reading). La intención en ambas era que se marcara el inicio de un cambio que permitiera que ese espacio fuera algo diferente de la biblioteca tradicional. El resto de casos han adoptado hasta cierto punto el concepto de $L C$ a través de extensiones y remodelaciones a lo largo de los años. Se pretendía proporcionar áreas para diversos tipos de aprendizaje: se añadieron en todas las bibliotecas estudiadas zonas de trabajo en grupo, de ordenadores, puntos de red para portátiles y aulas con facilidades informáticas. Aunque el director de la biblioteca de Birkbeck College afirmó que no tuvieron en mente este apoyo, los cambios realizados, de hecho, mejoraron la capacidad formativa: la instalación de la red inalámbrica o el aumento de ordenadores, por ejemplo. Es muy importante que en las reformas que se realicen subyazca la filosofía pedagógica. Esto se comprobó que existía en la biblioteca de Royal Holloway.

\section{"Es importante que en las reformas que se realicen exista una filosofía de enseñanza que las apoye"}

La biblioteca de la Universidad de Bath construyó un edificio, denominado Library and Learning Centre que estaba dividido por la mitad en sentido vertical: es decir la parte exterior de todas las plantas se dedicaba a los estudiantes y la del fondo a la investigación. Su di- rector exponía que al diseñarla tenían en mente el "modelo librería". Es decir, aplicaron criterios comerciales para hacer los servicios atractivos a los estudiantes y conseguir más alumnos.

Este edificio tiene cinco pisos y la idea era construir un $L C$ pero conservando el espacio para la investigación al mismo tiempo. Para esto han colocado los ordenadores y las instalaciones para el aprendizaje en la parte delantera de la biblioteca y las zonas de estudio en silencio y los materiales de investigación (revistas en papel, lectores de microformas, etc.) en la zona de atrás. Después de algunos años se han instalado mamparas acristaladas para favorecer el aislamiento de las zonas.

Los servicios generales se sitúan en el primer piso y parte del segundo. Hay zonas de trabajo en grupo en las plantas dos y cuatro. En total, hay cuatrocientos ordenadores divididos entre varios pisos; de hecho, la gran mayoría de los disponibles en la universidad están en la biblioteca. Además, en todo el edificio hay puntos de red para portátiles, donde los estudiantes pueden estudiar individualmente o en grupos, sentados en mesas, o en un entorno más informal, con sillones o taburetes, con la posibilidad de utilizar impresoras. Hay puestos de ayuda informática o soporte técnico en algunas plantas $\mathrm{y}$ en todo el edificio se pueden encontrar instalaciones para materiales audiovisuales, al igual que una clase equipada con ordenadores para uso de la biblioteca.

La biblioteca de la Universidad de Reading dedica parte de una planta de su edificio a una zona que no se llama LC, sino S@IL: Student access to independent learning (acceso del estudiante al aprendizaje independiente). Aunque el nombre es un acrónimo, se utiliza la metáfora de la navegación en el mar, que es el significado de sail. De hecho, el color dominante en esta zona es el azul, porque se concibió como un lugar donde el estudiante navega por el conocimiento. Allí se sitúan ciento cuarenta ordenadores, además de impresoras, escáneres, etc. Es un $L C$ situado dentro de la biblioteca. Hay también instalaciones para estudiantes discapacitados y posibilidad de préstamo de cámaras de vídeo, proyectores, grabadoras de audio, existen además salas equipadas con ocho a diez ordenadores, proyectores, retroproyectores, etc., donde los alumnos pueden desde

\begin{tabular}{|l|c|c|c|c|c|c|}
\hline & Bath & Reading & Sussex & Birckbeck & Royal Holloway & York \\
\hline Nuevo edificio & - & - & - & - & - & $\checkmark$ \\
\hline Ampliación & $\checkmark$ & - & $\checkmark$ & $\checkmark$ & $\checkmark$ & $\checkmark$ \\
\hline Remodelación & $\checkmark$ & $\checkmark$ & - & $\checkmark$ & - & $\checkmark$ \\
\hline Proyecto de edificio & $\checkmark$ & $\checkmark$ & - & - & - & $\checkmark$ \\
\hline
\end{tabular}

Tabla 1. Cambio de instalaciones en los últimos años 
recibir clases y hasta preparar presentaciones. Por otra parte, hay un local donde pueden encontrar apoyo del equipo pedagógico de la universidad.

\section{"Tener un $L C$ no es suficiente para aportar un buen apoyo a la enseñanza. Tiene que estar respaldado por la biblioteca"}

La mayoría de los entrevistados consideran que el solo hecho de tener un $L C$ únicamente no es suficiente para aportar un buen apoyo a la formación. Tiene que estar respaldado por la biblioteca y, sobre todo, por el compromiso de la institución hacia la mejora de la educación de los estudiantes. Sin embargo, se observa por las respuestas obtenidas, que los $L C$ que existen en dos de las bibliotecas estudiadas han mejorado radicalmente la enseñanza, proporcionando un entorno excelente para el desarrollo de la misma. Algunas desventajas podrían ser la necesidad cada vez mayor de ordenadores, y la pérdida del ambiente de trabajo, ya que algunos estudiantes vienen a la biblioteca para accesos rápidos a internet.

El director de la biblioteca de Bath indicaba que no habían previsto que el cambio de las instalaciones, aparte de aumentar el número de personas que acudía, lo modificara cualitativamente, pues llegaban para conectarse a internet, consultar el correo electrónico, etc. El director de la biblioteca de Reading expresaba la esperanza de que, aunque no fuera su primer objetivo, los estudiantes acabaran usando los servicios bibliotecarios.

Las bibliotecas se han convertido en una extensión del aula, con un espacio complejo y multifuncional. Los estudiantes necesitan diferentes espacios para el estudio, individual y en grupo; éstos deben estar alejados de aquellos para estudiar en silencio (Bennett, 2005), así como espacios con ordenadores para consultar la información electrónica e impresoras.

La planificación de la construcción del $L C$ exige no sólo pensar en lo material, sino en las personas que lo van a utilizar (Bennett, 2005; McDonald, 2004). Se requiere tener en cuenta cómo interactúan con las colecciones, las nuevas tecnologías y los servicios (McDonald, 2004). Cuando se planifica este tipo de edificios es aconsejable pedir opinión y conocer las necesidades de los principales participantes: los profesores, los estudiantes y el personal. Los bibliotecarios deberían liderar esta planificación teniendo en cuenta que "crear nuevos espacios para el aprendizaje es una oportunidad única para cambiar la cultura y las actitudes dentro de la organización y para influir en el modo en que el personal y los usuarios se comportan" (McDonald, 2004).

El enfoque centrado en las personas se mencionó también en las entrevistas: unificar en un único espacio lo que el estudiante necesita. Por ejemplo en la Universidad de Reading proyectan construir un edificio para servicios a los estudiantes que va a estar conectado con la biblioteca. De la misma forma, en algunas bibliotecas parte del personal informático trabajaba en el mismo edificio (Bath), o existían espacios compartidos con el personal de apoyo al aprendizaje (Reading y Royal Holloway). El que en el mismo edificio estén bibliotecarios, informáticos y especialistas en educación, constituye el núcleo del concepto de $L C$ y puede realizarse sin mucho esfuerzo si hay buena colaboración entre los distintos servicios.

Los estudiantes también quieren relacionarse en el entorno del $L C$ y en muchos casos se han incluido cafeterías en sus instalaciones. Por ejemplo, en la Glasgow Caledonian University se ha creado un Learning café, donde los estudiantes pueden trabajar en grupos con materiales de la biblioteca y con ordenadores, al mismo tiempo que toman bebidas (Howden, 2004).

El director de la biblioteca de la Universidad de Bath reconocía que existe una necesidad de espacio para relacionarse con los compañeros. Transmitía su sorpresa al comprobar que los estudiantes iban al $L C$ más de una vez al día y que la demanda de ordenadores crecía continuamente, a pesar de que ese campus es muy pequeño y los alumnos tienen conexión a internet en las residencias distantes sólo diez minutos de la biblioteca. La necesidad de relacionarse de los estudiantes pensó que podría cubrirse con un local en el mismo edificio, aunque separado; algo parecido a un cibercafé, que pudiera utilizarse al mismo tiempo como espacio para trabajo en grupo y para consultas rápidas de internet.

\subsection{Cambio de nombre a $L C$}

En los cuestionarios y entrevistas se planteaba si existía la necesidad de cambiar el nombre de biblioteca por $L C$ para transmitir así el cambio de enfoque de la biblioteca hacia la formación. De los seis directores entrevistados, sólo uno pensaba que cambiarlo por uno como centro de recursos para el aprendizaje o algo similar fuera positivo para una universidad enfocada a la investigación. El resto de los entrevistados declaraba que este cambio no era adecuado para sus bibliotecas. Lo positivo de ello es acentuar las necesidades de información de los estudiantes, que podían ser olvidadas. Una biblioteca que debe apoyar la investigación, si 
pierde esa denominación y acentúa sólo la parte docente podría enviar un mensaje incorrecto a los usuarios. Un entrevistado pensaba que era mejor cambiar la concepción de biblioteca desde el significado tradicional de libros, revistas, etc., al actual de portal de acceso al conocimiento, a través de las instalaciones y recursos electrónicos. En concreto, este director (Universidad de York) expresaba su idea de la siguiente forma: "la biblioteca significa todavía algo. Pienso que 'biblioteca' es una palabra importante, porque significa mucho más que libros. Creo que es mejor desarrollar el concepto en la mente de las personas que cambiar el nombre".

En resumen, sólo dos de las seis bibliotecas han modificado su nombre, y siempre han conservado el término biblioteca junto con el nuevo. En la literatura estudiada (Hines, 2003), se indica que el cambio de nombre se ha dado fundamentalmente en aquellas bibliotecas que han cambiado de edificio, y principalmente en las llamadas "nuevas universidades". Así pues, se puede deducir que en los centros más enfocados hacia la investigación, el cambio de nombre puede no ser adecuado porque, en cierto modo, se excluye el apoyo a la investigación que también se realiza.

Una experiencia interesante en los casos analizados ha sido la de aislar una planta para la formación, denominándola de otra forma, con lo que se evita eliminar el título de biblioteca. Más recientemente se ha realizado otra experiencia similar, separando esa zona incluso de la biblioteca y situándola en un edificio de soporte al estudiante. Los resultados han sido buenos, aunque tiene la contrapartida de que, conceptualmente, esa zona se aísle de los servicios bibliotecarios ${ }^{3}$.

\section{Cambio de estructuras organizativas y apoyo al aprendizaje} cios

\subsection{Convergencia y colaboración entre servi-}

En los cuestionarios se preguntaba a los directivos de bibliotecas si constituían una unidad separada o bien eran gestionados conjuntamente con otros servicios. Las respuestas fueron muy diversas y se aclararon en mayor medida en las entrevistas (tabla 2). En la mayoría no se había modificado su estructura de gestión en los últimos años, y constituían unidades separadas dentro de la universidad. Sin embargo, dos bibliotecas eran gestionadas conjuntamente con otros servicios: en un caso con los servicios informáticos, y en otro formaban una unidad con servicios informáticos, audiovisuales y otros (de teléfonos y archivo).

En todos los casos, las respuestas subrayan la independencia de la biblioteca como una unidad separada. Incluso en los dos centros en que son gestionadas conjuntamente, los entrevistados destacan la autonomía con otros servicios. Se deduce de estas afirmaciones que esta estructura común se ha llevado a cabo de un modo formal y desde una instancia superior.

La colaboración entre servicios ha aumentado notablemente en el nuevo entorno electrónico. Las bibliotecas colaboraban no sólo, como se preguntaba, con los servicios informáticos y los educativos, sino también con otros tales como el apoyo al estudiante, equipos de elaboración de páginas web, expertos legales, etc. Algunos de los encuestados señalaban que las relaciones a veces han cristalizado en una estructura formal, como un comité de servicios de información para coordinar el trabajo de servicios informáticos y biblioteca, o un comité coordinado para desarrollar las plataformas de aprendizaje virtual. Para un buen apoyo al aprendizaje, es esencial también la cooperación con los servicios educativos. El mejor ejemplo es la biblioteca de Royal Holloway, que trabaja muy estrechamente con el servicio pedagógico hasta el punto de que se ubican en el mismo edificio y realizan muchos proyectos juntos. En Reading, un miembro del personal que se encarga del apoyo a la formación ha abierto muchas oportunidades a la biblioteca. Aunque estos dos centros están gestionados conjuntamente con los servicios informáticos, hacen hincapié en la buena relación y proyectos que se han hecho gracias a la colaboración con las unidades pedagógicas.

En Reading, el establecimiento de un $L C$ ha facilitado una mejor relación con los servicios informáticos

\begin{tabular}{|l|c|c|c|c|c|c|}
\hline & Bath & Reading & Sussex & Birckbeck & Royal Holloway & York \\
\hline Convergencia & - & - & - & - & - & - \\
\hline $\begin{array}{l}\text { Gestionada conjuntamente } \\
\text { con otro servicio }\end{array}$ & - & - & - & - & $\checkmark(1)$ & - \\
\hline Separada & - & $\checkmark$ & $\checkmark$ & $\checkmark$ & - & $\checkmark$ \\
\hline Otra organización & $\checkmark(2)$ & - & - & - & - & - \\
\hline $\begin{array}{l}\text { (1) La biblioteca se ha unido con los servicios informáticos, servicios audiovisuales y otros (teléfonos y archivo). } \\
\text { (2) Parte del personal de servicios informáticos trabaja en la biblioteca para mantener los ordenadores situados en ese edificio. }\end{array}$ \\
\hline
\end{tabular}

Tabla 2. Estructura organizativa de las bibliotecas estudiadas 


\section{"Evidentemente, para conseguir un buen apoyo a la formación, es esencial también la cooperación con los servicios educativos"}

y educativos: "Uno de los desencadenantes (de la colaboración) ha sido el establecimiento de las instalaciones que denominamos S@il. Así, ahora tenemos en la biblioteca unas grandes instalaciones para el aprendizaje donde trabajamos estrechamente con los servicios informáticos y el personal docente".

Uno de los directivos entrevistados opinaba que era un beneficio fundamentalmente desde un punto de vista estratégico. Es decir, que los servicios informáticos y la biblioteca estuvieran gestionados conjuntamente era debido a una razón de efectividad y de dar un mejor servicio, pero no había sido pensado para mejorar el aprendizaje. El director de la biblioteca de la Universidad de Bath pensaba que las estructuras no eran tan importantes como el tener las personas apropiadas y el adecuado enfoque estratégico. Este hecho se comprobó en dos universidades con estructura de convergencia de servicios. En Reading el nuevo organigrama no había modificado ni la forma de trabajar ni los proyectos que se llevaban a cabo. En cambio, en Royal Holloway, donde no existe convergencia con los servicios educativos, ha cambiado la perspectiva y ahora tienen muchos proyectos en e-learning. Por lo tanto, aparte de las estructuras que se cambian desde instancias superiores, es importante que los directivos sepan cómo aprovechar las oportunidades de la nueva situación.

De estos resultados se deduce que la colaboración con los servicios puede ser más positiva que las estructuras convergentes, sobre todo si se han llevado a cabo de un modo formal. Particularmente conveniente parece la colaboración con unidades educativas, que pueden abrir grandes posibilidades a las bibliotecas.

\subsection{Colaboración con los departamentos acadé- micos}

Las bibliotecas estudiadas partían de una excelente colaboración con los departamentos académicos a tra- vés del sistema de bibliotecarios temáticos, que funciona especialmente bien en tres centros (Bath, Reading y York). En el primero, el director afirmaba que el bibliotecario temático en la práctica era parte del departamento. Tradicionalmente estos profesionales han sido una gran ayuda a la investigación. En los últimos años también están intentando colaborar en la docencia a través de cursos en competencias en información impartidos a los alumnos.

Esto se ha llevado a cabo muy desigualmente en las bibliotecas estudiadas. En todas ellas se impartían sesiones sobre competencias en información, sin embargo, estaban formalizadas sólo en las universidades de Royal Holloway y York, y hasta cierto punto en la de Bath. En York existía una asignatura complementaria que imparte formación en competencias en información junto con habilidades informáticas, denominada Iliad (Information literacy for all departments) y aunque es optativa los profesores la aconsejan. En la mayoría de los casos no están dentro del plan de estudios y por tanto no son obligatorias. En Serrano (2005) se recogen algunas experiencias de introducción de esta materia en el plan de estudios en universidades británicas y de EUA.

Las bibliotecas también han intentado impartir más formación de usuarios coordinada a través de los bibliotecarios temáticos. La mayoría de las sesiones de formación están dedicadas a las competencias en información.

En el año anterior a esta investigación (2003) las bibliotecas que tienen un porcentaje más alto de asistencia de estudiantes en las sesiones de competencias en información han sido York (97\%) y Royal Holloway (82\%) (tabla 3).

Las razones del éxito de las sesiones de formación en estas dos universidades podemos encontrarlas en el excelente desarrollo del sistema de bibliotecarios temáticos, y también en la adaptación de las sesiones a las necesidades de los departamentos, que se detectan más fácilmente debido a la continua relación existente entre los bibliotecarios y académicos. En la Universidad de York no se hubieran podido organizar tantas sesiones prácticas si no hubieran creado lo que llaman el "Equipo de apoyo a los bibliotecarios temáticos", que consiste en un grupo de bibliotecarios que trabajan en

\begin{tabular}{|l|r|r|r|r|r|r|}
\hline & Bath & Reading & Sussex & Birckbeck & Royal Holloway & York \\
\hline Asistentes a sesiones & 5.400 & 6.074 & 2.043 & 252 & 4.954 & 8.810 \\
\hline Número total de estudiantes & 8.000 & 12.500 & 9.100 & 6.000 & 6.000 & 9.000 \\
\hline Porcentaje (\%) & 67 & 48 & 22 & 4 & 82 & 97 \\
\hline
\end{tabular}

Tabla 3. Asistentes a sesiones de formación durante el año 2003 
departamentos técnicos (catalogación, publicaciones periódicas, etc.), pero que cuando se necesita, apoyan a los bibliotecarios temáticos. Este hecho ha permitido que a una misma sesión acudan varios de ellos que orientan a los usuarios en las búsquedas, favoreciendo una ayuda más personalizada.

A lo largo del estudio se comprobó que una forma de mejorar el apoyo al aprendizaje de los alumnos es el sistema de bibliotecarios temáticos. Las razones para ello son variadas. En primer lugar, los profesores y bibliotecarios pueden intercambiar experiencias sobre el modo de mejorar la formación de los alumnos. Los bibliotecarios temáticos, por otra parte pueden informar a los profesores sobre los materiales existentes. Y por último, el mejor modo de ayudar a los alumnos es hacerlo con expertos en búsqueda de información, y para conseguir este objetivo es fundamental transmitir a los profesores la necesidad de impartir cursos obligatorios de competencias en información para estudiantes, consiguiendo de esta forma mejorar la calidad del aprendizaje independiente. Los bibliotecarios temáticos pueden conseguir estos propósitos si tienen una buena relación con los departamentos y son valorados por los académicos. Algunos de los entrevistados indican que no hay que limitarse a los contactos formales, en los que se informa de los recursos que la biblioteca tiene en una materia determinada, o se les orienta en la adquisición de materiales, sino que es muy conveniente también mantener contactos informales con ellos.

Como se ha afirmado respecto al edificio, también en este aspecto hace falta un apoyo institucional para conseguir este objetivo.

\section{Servicios}

\subsection{Aumento de servicios}

La encuesta que se realizó a los directivos reflejaba que estas bibliotecas habían aumentado sus servicios para apoyar el aprendizaje en los últimos años. Por supuesto, aquellas que habían instalado un $L C$ aumentaron los recursos, pero también lo habían hecho las que mantenían unos servicios más tradicionales: áreas de ordenadores, zonas de trabajo en grupo, materiales audiovisuales, amplios horarios de apertura, mejores recursos electrónicos de información y de aprendizaje, mejores cursos de formación de usuarios y servicios para discapacitados (tabla 4).

Es llamativa la irregularidad de la provisión de las zonas de trabajo en grupo. En una universidad sólo existe una sala de trabajo en grupo, y no se usa mucho y, en contraste, hay doce salas en otra biblioteca, con una demanda como para treinta más. Este hecho está muy relacionado con la utilización del método de trabajo en grupo por parte de la universidad: si ésta lo usa, entonces la biblioteca debe proveer espacios para los alumnos, generalmente con ordenadores o al menos con conexiones inalámbricas. Uno de los directivos tenía la idea de poner conexión inalámbrica en espacios públicos, como las cafeterías, con el objetivo de tener más espacios de trabajo en grupo, considerando que así se podía resolver la falta de espacio para trabajo en grupo y también reducir el número de usuarios que va a la biblioteca para un uso rápido de internet. Esta solución sería mejor si las cafeterías se situaran dentro de la biblioteca, donde los alumnos pudieran acceder a los fondos bibliográficos.

En todos los casos existían mediatecas y materiales audiovisuales. En las visitas que se realizaron se apreció una mejor provisión de materiales audiovisuales en la Universidad de Reading.

Respecto a las horas de apertura, la mayoría de las bibliotecas habían aumentado sus horarios, aunque sólo la de la Universidad de Bath abría 24 horas. Dos de los directivos entrevistados opinaban que abrir los servicios informáticos todo el día era suficiente para cubrir las necesidades de los estudiantes durante la noche. De acuerdo con las webs de las bibliotecas estudiadas, la media de horas de apertura era de 13 de lunes a jueves, 11 los viernes y 7 los fines de semana. El horario de cierre oscilaba entre las 10 y las 11 de la noche. Uno de los directivos acentuaba el hecho de que ahora abren los fines de semana el doble de lo que solían. Por otra parte, en la biblioteca de Bath, que, como se ha dicho, abre 24 horas, tenían algunas complicaciones como el mantenimiento del mobiliario, de comida y bebida, y lo que denominaban "problemas de seguridad": existían personas que parecían vivir en la biblioteca. Conviene tener en mente estas cuestiones a la hora de tomar una decisión.

Por otra parte, de acuerdo con una encuesta, Ward (2003) señala que los estudiantes acuden por la noche principalmente para escribir ensayos, buscar bibliografía, imprimir recursos electrónicos y usar el correo electrónico e internet. De estas actividades, solamente buscar bibliografía no puede ser totalmente completado desde internet, porque no se puede acceder a los materiales impresos. Sin embargo, si la biblioteca tiene una buena colección electrónica, puede ser suficiente para las necesidades de los estudiantes.

Por lo tanto, los materiales disponibles electrónicamente (libros, revistas, etc.) parecen especialmente importantes para el apoyo al aprendizaje (tabla 5). Además, las bibliotecas intentan hacer accesibles materiales que hasta ahora sólo se encontraban en formato impreso, como la de Royal Holloway que puso 100 guías de la biblioteca a través de internet así como un tutorial de introducción. Por otra parte, la digitalización de ma- 


\begin{tabular}{|l|c|c|c|c|c|c|}
\hline & Bath & Reading & Sussex & Birckbeck & Royal Holloway & York \\
\hline Trabajo en grupo & $\checkmark$ & $\checkmark$ & $\checkmark$ & $\checkmark$ & $\checkmark$ & $\checkmark$ \\
\hline Ordenadores & $\checkmark$ & $\checkmark$ & $\checkmark$ & $\checkmark$ & $\checkmark$ & $\checkmark$ \\
\hline Impresoras & $\checkmark$ & $\checkmark$ & $\checkmark$ & $\checkmark$ & $\checkmark$ & - \\
\hline Zonas de estudio individual & $\checkmark$ & $\checkmark$ & $\checkmark$ & $\checkmark$ & $\checkmark$ & $\checkmark$ \\
\hline $\begin{array}{l}\text { Materiales e instalaciones para } \\
\text { audiovisuales }\end{array}$ & $\checkmark$ & $\checkmark$ & $\checkmark$ & $\checkmark$ & $\checkmark$ & $\checkmark$ \\
\hline Conexiones a internet para portátiles & $\checkmark$ & $\checkmark$ & $\checkmark$ & - & $\checkmark$ & - \\
\hline Apertura 24 horas & $\checkmark$ & - & - & - & $\checkmark$ & - \\
\hline Aulas para sesiones de formación clases & $\checkmark$ & $\checkmark$ & $\checkmark$ & $\checkmark$ & $\checkmark$ & $\checkmark$ \\
\hline $\begin{array}{l}\text { Áreas donde los estudiantes pueden } \\
\text { solicitar orientación pedagógica }\end{array}$ & - & $\checkmark$ & - & - & - & $\checkmark$ \\
\hline
\end{tabular}

Tabla 4. Servicios relacionados con LCs existentes en las bibliotecas estudiadas

\begin{tabular}{|l|c|c|c|c|c|c|}
\hline & Bath & Reading & Sussex & Birckbeck & Royal Holloway & York \\
\hline Programas de aprendizaje & $\checkmark(1)$ & - & - & - & $\checkmark$ & - \\
\hline Formación en alfin & $\checkmark(2)$ & $\checkmark$ & $\checkmark(3)$ & $\checkmark$ & $\checkmark$ & - \\
\hline Ninguno & - & - & - & - & & - \\
\hline $\begin{array}{l}\text { (1) Disponibles en la web de la universidad. } \\
\text { (2) A nivel muy básico. } \\
\text { (3) Desde octubre 2004. }\end{array}$
\end{tabular}

Tabla 5. Materiales de estudio disponibles en la web

teriales es una de las formas de poner disponibles los recursos de aprendizaje y también los fondos antiguos.

Las bibliotecas están trabajando a diferentes niveles los entornos virtuales de formación. En Reading y Royal Holloway, la unidad de apoyo docente está colaborando estrechamente con la biblioteca en la implementación de WebCT y Blackboard. En la primera, un miembro del personal se ha nombrado coordinador del soporte al aprendizaje. En la segunda, se apoya al personal académico en los cursos online y en los entornos virtuales de aprendizaje y además la unidad responsable está situada en el edificio de bibliotecas. En el resto de las universidades estudiadas los entornos de enseñanza virtual están menos evolucionados y las bibliotecas están implicadas en diferentes proporciones. La de la Universidad de York formaba parte del comité de realización de la plataforma de enseñanza virtual, y de momento estaban digitalizando recursos para estar preparados cuando la plataforma sea utilizada más ampliamente en el campus.

Por último, los entrevistados indican que los servicios para discapacitados han aumentado en los últimos años; especialmente en Reading se encuentran varias opciones, como ampliar las letras para personas con deficiencias visuales u otros componentes de auxilio para sordos. En la Universidad de Sussex hay un bi- bliotecario para usuarios que tengan unas necesidades especiales.

\subsection{Retos y problemas en el desarrollo de nue- vos servicios}

Las respuestas se agrupan según los problemas que se expusieron.

- Económicos: alto coste de los recursos electrónicos, necesidad de cambio de mobiliario debido al mayor uso, demanda creciente de ordenadores, contratación de más personal para abrir 24 horas.

- Problemas de comportamiento de los usuarios.

- Las bibliotecas se han visto superadas por la mayor cantidad y la naturaleza diversa de los estudiantes, debido a los cambios en la educación superior que se han dado últimamente en el Reino Unido.

- Falta de entendimiento entre los investigadores y el personal de la biblioteca cuando se han adoptado nuevos servicios.

- Colaboración con los servicios informáticos en el mantenimiento de los ordenadores que se han situado en la biblioteca.

- Necesidad de formación de investigadores y bibliotecarios en el contexto electrónico. 
En conclusión, el nuevo entorno electrónico debido al paso de la biblioteca tradicional a los $L C \mathrm{~s}$, con los cambios radicales que se han llevado a cabo, ha creado una serie de problemas que no existían antes. Se requiere un esfuerzo del equipo de dirección de la biblioteca para mantener los objetivos transmitiendo la idea apropiada de lo que debería ser un Centro de Recursos para el Aprendizaje. Como indicaba el directivo de la biblioteca universitaria de Reading, eso implica entablar muchas relaciones públicas para hacer entender a los profesores y al personal de la biblioteca la nueva política.

\section{3. $L C \mathrm{~s}$ y apoyo a la investigación}

Una de las cuestiones planteadas en este estudio era cómo se podían hacer compatibles en un mismo edificio las necesidades de espacio para el aprendizaje y la investigación. Recordemos que se estudiaban universidades de mediano o pequeño tamaño y que en el Reino Unido existe un solo edificio como biblioteca para estudiantes y profesores. Por lo tanto, es necesario cubrir en menos espacio las necesidades de ambos tipos de usuarios. La pregunta planteada a los directivos se refería a si los cambios en el edificio y los servicios para mejorar el aprendizaje habían afectado al uso que hacían los investigadores por una parte, y si las necesidades de información de profesores y estudiantes eran compatibles en un mismo edificio. Por último, se preguntaba si el $L C$ ayudaba a centrarse en los estudiantes en universidades muy enfocadas a la investigación.

Los nuevos recursos e instalaciones han afectado a los investigadores en todas las universidades estudiadas. Dos consideran que la mejora ha beneficiado a los investigadores, especialmente el aumento de medios electrónicos y la ampliación de horarios de apertura. También ha sido beneficioso para ellos el aumento de la provisión de materiales para la enseñanza en la biblioteca.

Sin embargo, un problema que se ha planteado es el ruido que se produce en las zonas de trabajo en grupo y ordenadores. Los investigadores protestan porque no pueden estudiar en silencio, y algunos han dejado de trabajar en la biblioteca. La solución que se ha dado en la literatura para conservar zonas de trabajo en silencio es separar las diferentes actividades en distintas áreas (McDonald, 2004; Hines, 2003). En la Universidad de Bath se trató de separar por zonas las actividades, mediante mamparas de cristal, pero no se han solucionado todos los problemas, porque al tener dividida la biblioteca en vertical, es difícil hacer comprender a los alumnos que pueden hablar en una zona de la planta y no en la otra. Dos de las universidades (Royal Holloway y York) han resuelto el problema del ruido porque el personal controla las áreas de estudio en silencio.
Otra solución es colocar las áreas de trabajo en grupo en un piso o local diferente. También se sugieren otras opciones, como utilizar en la construcción del edificio materiales absorbentes del ruido (McDonald, 2004).

Las nuevas instalaciones dedicadas al aprendizaje necesitan mucho más espacio. Cuando no se ha construido un nuevo edificio, sino adaptado el que ya se tenía, parte del espacio dedicado a la investigación se ha utilizado para instalar los recursos de aprendizaje. Por ejemplo, en Reading se han eliminado muchas de las revistas para instalar el $L C$. Sin embargo, más adelante se dieron cuenta que la zona dedicada a la investigación era muy pequeña. En la actualidad van a construir un edificio para fondo antiguo que será utilizado como biblioteca de investigación.

La solución que parece más adecuada es tener diferentes espacios para la variedad de usuarios.

Sólo uno de los seis entrevistados consideraba necesario tener un $L C$ para un buen apoyo a la formación. Tres de ellos indicaban que una universidad dedicada a la investigación podía ser brillante en la enseñanza, de la misma forma que una buena biblioteca de investigación con los recursos educativos apropiados, podía ser realmente de ayuda para los estudiantes. Es decir, un centro bien surtido de materiales de investigación podía apoyar al aprendizaje en gran medida si estaba correctamente surtido también de materiales docentes. Recogemos las respuestas de estos entrevistados, que parecen muy interesantes:

- "Si la institución está ya suficientemente centrada en la enseñanza y además tiene éxito en la investigación, entonces el LC no va a marcar la diferencias. En parte esto es porque existe un prejuicio de que los investigadores no enseñan bien, cuando de hecho algunos investigadores son brillantes dando clases, y algunos no, y algunas instituciones de investigación dan a los estudiantes una gran formación" (Universidad de Bath).

- "Pienso que no es fácil decir dónde terminan las necesidades de un estudiante y dónde empiezan las de un postgraduado. Para un estudiante es mejor utilizar un amplio rango de revistas y visitar otras bibliotecas en Londres, no sólo ésta, y estar involucrado en proyectos de investigación" (Universidad Royal Holloway).

- "Porque queremos que nuestros estudiantes vean y compartan la colección de investigación y que se entusiasmen con la actividad investigadora, de tal modo que puedan decir: quiero convertirme en un investigador. Estoy apasionada con este tema" (York).

Para una universidad orientada a la investigación siempre será necesaria una biblioteca donde se pueda realizar este trabajo, especialmente para aquéllos que 
se dedican a la investigación en humanidades. Deegan (1998) indica que la biblioteca de investigación es la identidad para la universidad orientada a la misma, y para los investigadores en humanidades es su laboratorio. Aunque puedan acceder a muchos de los materiales electrónicamente (nunca podrán llegar a todo), siempre necesitarán ese espacio que les permita estudiar en la biblioteca. Por otra parte, los entrevistados piensan que la mejor herencia que pueden dejar los profesores en los alumnos es enseñarles las técnicas de investigación. Para eso es necesaria una biblioteca que tenga buenos materiales y servicios de apoyo a la investigación.

\section{Conclusiones}

Los LCs mejoran el aprendizaje, aunque se ha comprobado que se necesita también una actitud de apoyo por parte de la biblioteca y el compromiso de la institución. La experiencia nos dice que han atraído a un mayor número de estudiantes, más con el objetivo de utilizar las instalaciones y ordenadores que para el uso tradicional de las bibliotecas. Por lo tanto se ha producido un cambio cualitativo en los usuarios. El enfoque centrado en las personas es fundamental a la hora de planificar el $L C$. Por esta razón es aconsejable que en el espacio dedicado a biblioteca se sitúen otros servicios que puedan completar las necesidades de los alumnos. Se ha comprobado que existen casos de inclusión de servicios informáticos o pedagógicos en el mismo edificio, y también de otros de carácter administrativo que pueden ser de utilidad para los estudiantes.

\section{"Es aconsejable que en el espacio dedicado a biblioteca se sitúen otros servicios que puedan completar las necesidades de los alumnos"}

La experiencia de un $L C$ como parte del edificio ha tenido éxito en las universidades medianas enfocadas a la investigación. Por ejemplo, una planta dentro de un edificio o una zona aislada dentro de la biblioteca permiten tener diferentes áreas para los distintos tipos de usuarios: estudio en silencio, trabajo en grupos, trabajo con ordenador, etc.

Los cambios en estructuras han sido frecuentes en las universidades británicas a lo largo de los últimos años, y seguirán evolucionando (Corrall, 1995). Muchas bibliotecas universitarias británicas se han unido con diferentes servicios, pero los que han experimentado una mejora de las actividades de enseñanza y aprendizaje han sido aquéllas en las que se ha llevado a cabo una convergencia con unidades educativas (Oyston, 2003b). La colaboración con otros servicios, y especialmente con los departamentos académicos, parece muy útil para mejorar el rendimiento pedagógico (Sutton, 2000; Day; Edwards, 1998).

En este estudio destaca el hecho de que la convergencia impuesta de un modo formal no ayuda más a la enseñanza que una buena relación con otros servicios. De hecho, se llega a la conclusión de que dicha colaboración es el mejor modo de apoyar la formación. Las bibliotecas desean mantener su independencia, pero en el nuevo entorno electrónico no se puede lograr nada sin un esfuerzo conjunto de diferentes servicios de la universidad. Es necesaria una buena cooperación entre los servicios informáticos y los de innovación educativa. En algunas universidades incluso tienen personal pedagógico dentro de la biblioteca.

El enfoque $L C$ puede tanto beneficiar como marginar a los investigadores en las bibliotecas universitarias. Aunque los centros de recursos han resultado muy beneficiosos para el estudiante, han afectado negativamente hasta cierto punto a los investigadores. El apoyo al aprendizaje ocupa mucho espacio de la biblioteca y en ocasiones ha reducido el espacio para investigación. Por otra parte, existe el problema del ruido causado por el murmullo en zonas de trabajo en grupo, y de ordenadores. Se han adoptado algunas soluciones para este problema, como la de dividir en zonas las actividades, llegándose a la conclusión de que lo mejor es situar las áreas de trabajo en grupo y en silencio en distintas plantas.

En el nuevo entorno electrónico las bibliotecas universitarias tienen el reto de cubrir tanto las necesidades de los estudiantes como de los investigadores, en un espacio a veces más reducido de lo que se desearía.

\section{Notas}

1. No se ha utilizado el término CRAI (Centros de recursos para el aprendizaje y la investigación), porque en este estudio interesaba distinguir entre el espacio destinado al aprendizaje y del de apoyo a la investigación.

2. Las personas entrevistadas fueron:

Bath: Howard Nicholson, director

Birkbeck College: Philipa Dolphin, directora

Reading: Julia H. Munro, directora

Royal Holloway: David Ward, subdirector

Sussex: Maggie Fieldhouse, subdirectora

York: Elisabeth Heaps, directora

Los directivos mencionados de Birkbeck, Royal Holloway y Sussex no están en la actualidad en dichos centros.

3. Learning Grid (University of Warwick).

\section{Bibliografía}

Bennett, S. "Righting the balance". En: Library as a place: rethinking roles, rethinking space. Washington: Council of Library and Information Resources, 2005. ISBN 1-932326-13-8. Consultado en: 17-04-07.

http://www.clir.org/PUBS/reports/pub129/pub129.pdf 
Beton, H. An investigation of management issues arising from the transition from academic libraries to learning centres. Sheffield: M. A., University of Sheffield, 1999 [No publicado].

Brophy, P. The academic library. London: Library Association, 2000. ISBN 1856043746

Brophy, P. The library in the twenty-first century: new services for the information age. London: Library Association Publishing, 2001. ISBN 1856043754 .

Bulpitt, G. "The Learning Centre model in the UK. The Learning Centre in practice". En: I Jornadas Rebiun, 2003. Consultado en: 14-04-07. http://biblioteca.uam.es/documentos/Jornadas_REBIUN/1\%20-\%20lc_ \%20model.ppt

Corrall, S. "An evolving service: managing change". En: Lorcan Dempsey, Derek Law, Ian Mowat (eds.). Networking and the future of libraries: managing the intellectual record. London: Library Association Publishing, 1995, pp. 45-61. ISBN 1856041581.

Day, J.; Edwards, C. "Overview: managing change". En: Hanson, T.; Day, J.(eds.). Managing the electronic library: a practical guide for information professionals. London: Bowker-Saur, 1998, pp. 129-152. ISBN 1857391845.

Deegan, M. "The electronic library in teaching and research". En: Hanson, T.; Day, J.(eds.). Managing the electronic library: a practical guide for information professionals. London: Bowker-Saur, 1998, pp. 3-24. ISBN 1857391845.

Edwards, B.; Fisher, B. Libraries and Learning Resource Centres. Oxford: Architectural Press, 2002, ISBN 0750646055.

Hines, H. (ed.). "Building new environment: the physical space". En: Oyston, E. (ed.). Centred on learning: academic case studies on learning centre development. Hants: Ashgate, 2003, pp. 153-180. ISBN 0754608050

Howden, J. "Real@Caledonian: the Learning Café". En: Serials, 2004, v. 17, n. 1, pp. 15-18.

Joint Funding Councils' Libraries Review Group Report (Follett Report), Chair: Professor Sir Brian Follett, Bristol: Hefce, 1993. Consultado en: 1705-07.

\section{http://www.ukoln.ac.uk/services/papers/follett/report/}

Martin, D. "Pioneering design for 21st century learning: Learning Resources Centres at the University of Hertfordshire". En: II Jornadas Rebiun, 2004

McDonald, A. "Creating learning resource centres for the future: some qualities and reflections". En: II Jornadas Rebiun, 2004.

National Committee of Inquiry into Higher Education (Ncihe). Higher education in the Learning Society (Dearing Report). Bristol: HMSO, 1997.

Naylor, B. “'Old' Universities”. En: Baker, D. (ed.). Resource management in academic libraries. London: Library Association Publishing, 1997, pp. 37-49. ISBN 1856040364

Oyston, E. "Introduction". En: Oyston, E. (ed.). Centred on learning. academic case studies on Learning Centre Development. Hants: Ashgate, 2003a. ISBN 0754608050

Oyston, E. (ed.). "Organizational frameworks". En: Oyston, E. (ed.). Centred on learning: academic case studies on Learning Centre Development. Hants: Ashgate, 2003b, pp. 19-56. ISBN 0754608050.

Serrano, R. "Alfabetización en información en bibliotecas universitarias. Algunas experiencias en el ámbito anglosajón". En: Fesabid 2005 Infogestión: 9as. Jornadas Españolas de Documentación Documat. Madrid: Fesabid, 2005, pp. 509-519. ISBN 84-930335-5-3.

Sutton, A. "Convergence: a review of the literature". En: Achieving cultural change in networking libraries. Aldershot: Gower, 2000, pp. 63-75. ISBN 0566082004.

Ward, A. (ed.). "The student experience". En: Oyston, E. (ed.). Centred on learning: academic case studies on Learning Centre Development. Hants: Ashgate, 2003, pp. 97-126. ISBN 0754608050.

Rocío Serrano Vicente, subdirectora del Servicio de Bibliotecas, Universidad de Navarra, Biblioteca de Ciencias, Pamplona.

rserranov@unav.es

\section{Te damos los ingredientes...}
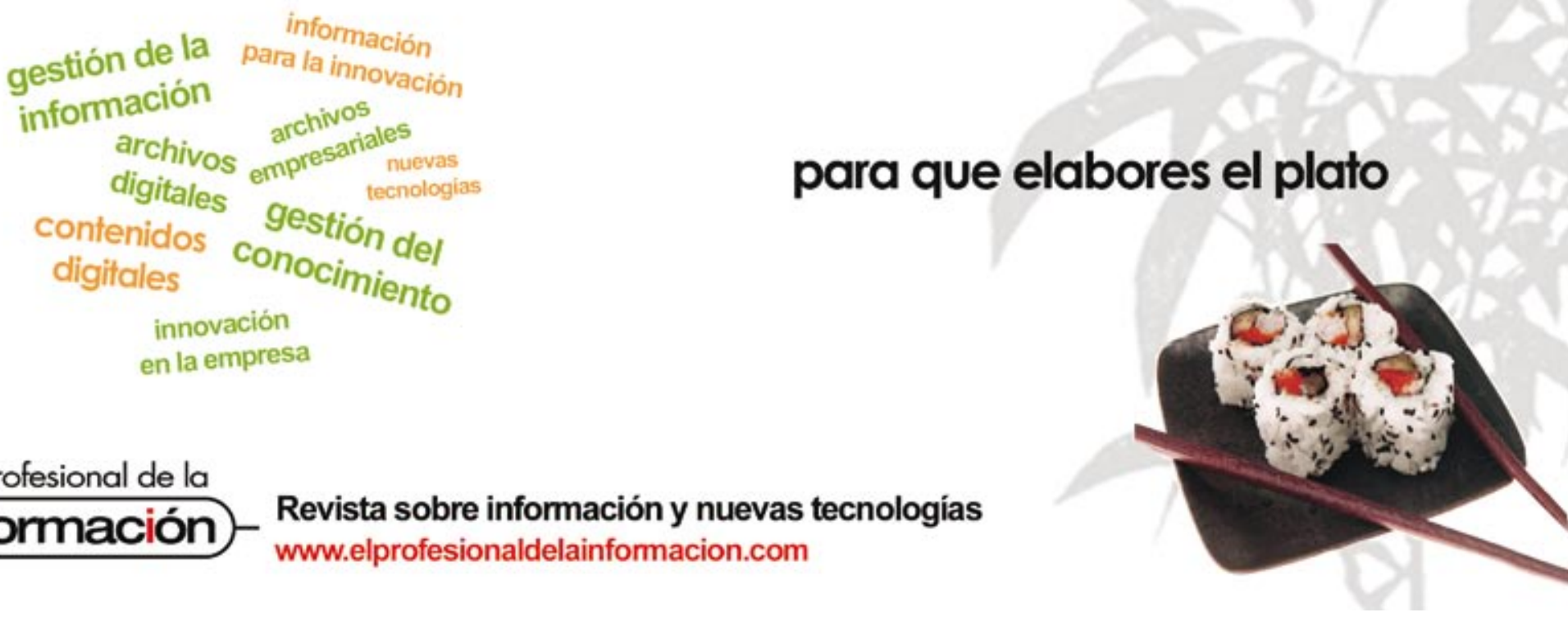